\title{
Erratum to: The Influence of the Numerical Aperture of a Beam Probing an Object on the Determination of the Thickness of a Layered Object in Confocal Microscopy
}

\author{
D. V. Lyakin ${ }^{a, *}$, L. A. Maksimova ${ }^{a}$, A. Yu. Sdobnov ${ }^{a}$, and V. P. Ryabukho ${ }^{a}$ \\ ${ }^{a}$ Institute of Precision Mechanics and Control, Russian Academy of Sciences, Saratov, 410028 Russia \\ *e-mail:LDV-77@mail.ru \\ Received June 18, 2019
}

DOI: $10.1134 /$ S0030400X19090078

Formula (14) should read

$$
k_{z 0}=\left(k_{z \max }+k_{z \min }\right) / 2=\frac{\pi}{\lambda_{01}} n_{0}\left(1+\cos \left(\theta_{\max }\right)\right) .
$$

Formula (16) should read

$$
\theta_{\text {eff }}=\arccos \left(\frac{\lambda_{01}}{2 \pi n_{0}} k_{z 0}\right)=\arccos \left(\frac{1}{2}\left(1+\cos \left(\theta_{\max }\right)\right)\right) .
$$

Formula (17) should read

$$
N A_{\text {eff }}=n_{0} \sin \left(\theta_{\text {eff }}\right)=\frac{1}{2} \sqrt{2 N A^{2}+\left(n_{0}-\sqrt{n_{0}^{2}-N A^{2}}\right)^{2}} .
$$

The original article can be found online at https://doi.org/10.1134/S0030400X17090235 